\title{
Modélisation du réseau trophique pélagique et de la production primaire en Manche
}

\author{
Thierry HOCH * \\ Ifremer, centre de Brest, Del - BP 70, 29280 Plouzané, France \\ IUEM, laboratoire d'océanographie chimique, université de Bretagne occidentale, technopôle Brest-Iroise, place Nicolas- \\ Copernic, 29280 Plouzané, France \\ * Adresse pour correspondance : Inra, centre de Clermont-Theix, 63122 Saint-Genes-Champanellc, France
}

(Revisé le 25 septembre 1998, accepté le 12 octobre 1998)

\begin{abstract}
Modelling pelagic food web and primary production in the English Channel. This paper deals with a model of the pelagic ecosystem in the English Channel. The model takes into account nitrogen biogeochemical cycle explicitly and indirectly phosphorus, silicon and carbon ones. The pelagic ecosystem is described precisely, on the one hand with the help of three phytoplanktonic compartments, and the other by considering the microbial food web. Primary production appears to be mainly controlled by stratification and turbidity, except in the Bay of Seine where high terrestrial nutrient inputs generate a sharp enrichment. Moreover, the model allows some general ecological conclusions to be drawn. From the simulation, it appears that inorganic nitrogen in the water column is mainly produced by an intense recycling. In accordance with in situ measurements, these results tend to demonstrate the predominance of the regenerated production over the new one in the English Channel. Simulation of nitrogen fluxes on an annual basis underlines the importance of bacterial remineralization, underestimating microzooplanktonic excretion in the recycling of elements. Furthermore, exchanges between pelagic and benthic compartments appear to be weak. $(\mathcal{C}$ Elsevier, Paris
\end{abstract}

\section{English Channel / ecological model / primary production / nitrogen fluxes}

Résumé - Cet article présente un modèle de la production pélagique en Manche. Il prend en compte explicitement le cycle biogéochimique de l'azote et, de manière indirecte ceux du phosphore, du silicium et du carbone. L'écosystème pélagique est décrit de manière précise, d'une part à l'aide de trois compartiments phytoplanctoniques, d'autre part par la prise en compte de la boucle microbienne. La production primaire apparaît essentiellement contrôlée par la stratification et la turbidité, sauf en baie de Seine où les forts apports terrigènes engendrent un enrichissement prononcé. Le modèle permet de dégager des conclusions ćcologiques de portée générale. Ainsi, d'après la simulation, l'azote inorganique dans la colonne d'eau est produit principalement par un recyclage intense. Confirmés par les mesures in situ, ces résultats montrent la prédominance de la production régénérée sur la production nouvelle en Manche. La simulation des flux d'azote à l'échelle annuelle souligne l'importance de la reminéralisation par les bactéries, sous-estimant sans doute l'excrétion par le microzooplancton dans le recyclage des éléments. Les échanges entre les compartiments pélagique ct benthique sont faibles. (C) Elsevier, Paris

\section{Manche / modèle écologique / production primaire / flux d'azote}

\section{INTRODUCTION}

Les zones côtières jouent un rôle non négligeable dans la production biologique des océans. Bien qu'elles ne représentent que $8 \%$ de la surface des océans, elles fournis- sent environ le quart du carbone organique marin [23] et $80 \%$ des ressources vivantes marines de la planète y sont exploitées. Les mers épicontinentales, à l'interface «océan-continent», constituent la zone d'impact des activités humaines. L'influence croissante de l'homme 
sur les écosystèmes côtiers en fait des sites d'étude privilégiés.

L'étude de ces zones nécessite la mise en place d'outils offrant une vision synoptique de la production biologique, et plus particulièrement primaire. La modélisation écologique à mésoéchelle permet de simuler et de synthétiser la dynamique de l'écosystème, et de tester l'influence de divers facteurs tels que les apports par les fleuves ou les variations météorologiques. Un tel modèle a été appliqué à la Manche dans le cadre du Programme national d'océanographie côtière (PNOC) pour plusieurs raisons : (1) cette mer située à la transition entre l'océan Atlantique et la mer du Nord est sujette à des phénomènes récurrents d'eutrophisation, (2) la Seine y déverse des quantités importantes d'éléments nutritifs et de polluants, (3) la structure physique de la Manche est bien connue et modélisée [34]. Un modèle de ce type a été précédemment appliqué à la Manche, d'une part afin de décrire l'effet de la structure verticale des masses d'eau sur la production primaire [25], d'autre part pour analyser la sensibilité du système, notamment aux conditions météorologiques et aux apports par les fleuves [11]. Ces travaux ne concernaient que les cycles biogéochimiques de l'azote, du phosphore et du silicium. Le présent travail inclut l'élément carbone au modèle, afin de donner une vision globale de la production primaire carbonée et de connaître son déterminisme. Les travaux précédents ne tenaient pas compte du broutage du phytoplancton par l'intermédiaire des hétérotrophes, ni de la production primaire induite par les cellules de très petites tailles, appelées ici nanoflagellés. L'importance quantitative des nanoflagellés a été mise en évidence dans des régions bien stratifiées de Manche occidentale par Holligan et al. [13]. Le nanoplancton doit être pris en compte dans l'étude de la production primaire carbonée en Manche. Vézina et Platt [36] ont utilisé ces mêmes données pour estimer les stocks et les flux dans un réseau trophique théorique, à l'aide d'une méthode inverse. Ils soulignent le rôle central du microzooplancton, des protozoaires et des bactéries dans le processus de recyclage au sein de la couche de surface. L'étude des flux de matière dans le réseau trophique pélagique nécessite donc la formulation explicite du recyclage des éléments dans la boucle microbienne.

Ce travail a pour objectif l'étude de la production primaire carbonée en Manche, à l'aide d'un modèle des composantes autotrophes et hétérotrophes de l'écosystème pćlagique.

\section{UN MODÈLE DE L'ÉCOSYSTÈME PÉLAGIQUE APPLIQUÉ À LA MANCHE}

\subsection{Simulation de la structure physique de la Manche}

Un tel modèle résulte du couplage entre un sous-modèle physique et un sous-modèle biologique. Menesguen et Hoch [25] ont simulé la structure physique de la Manche, divisée en 71 boîtes entre lesquelles les flux d'eau sont calculés à l'aide du logiciel Élise [24], à partir d'un modèle de circulation à mailles fines [34] qui reproduit le mouvement général des masses d'eau de l'ouest vers l'est. La structure verticale est simulée à l'aide du modèle intégral bicouche thermohalin, élaboré par Niiler et Kraus [27], puis repris par Agoumi et al. [1]. Il fournit pour chaque couche la température, la salinité et les vitesses d'échange entre la surface et le fond qui seront appliquées à tout constituant libre de la colonne d'eau.

La simulation est en accord avec les mesures : une stratification thermique apparaît au printemps en Manche occidentale et la thermocline sépare distinctement les couches de surface et de fond jusqu'au mois d'octobre. $\grave{A}$ l'embouchure de la Seine, une halocline stable est simulée, principalement en hiver, lorsque les débits d'eau douce sont maximaux.

\subsection{Modélisation du cycle des éléments}

\subsubsection{Conception du modèle et formulation des processus}

Hoch et Ménesguen [11] ont modélisé les cycles de l'azote, du phosphore et du silicium en considérant ces éléments sous les formes suivantes :

- inorganique dissoute,

- phytoplanctonique, comprenant diatomées et dinoflagellés, la croissance de ces derniers étant simulée par un modèle à quotas,

- détritique dans l'eau,

- organique benthique.

Nous allons détailler les modifications apportées ici à ce premier modèle. Painting et al. [28] ont montré l'importance du nanoplancton dans le bilan de la production primaire d'une zone d'upwelling au large de l'Afrique du Sud. Ils s'appuyent sur un modèle élaboré par Moloney et Field [26] qui considèrent trois classes de taille pour les autotrophes, et quatre pour les hétérotrophes. BarettaBekker et al. [4] ont montré que l'on pouvait simuler de manière réaliste la dynamique d'un système planctonique 
en mésocosme, à l'aide d'un modèle de la boucle microbienne appliqué à l'échelle de la mer du Nord. Le modèle développé dans le cas présent s'inspire de ces travaux. ainsi que du modèle de l'écosystème pélagique marin développé par Andersen et Rassoulzadegan [2] et appliqué à la Méditerranée. La figure $I$ résume les idées qui

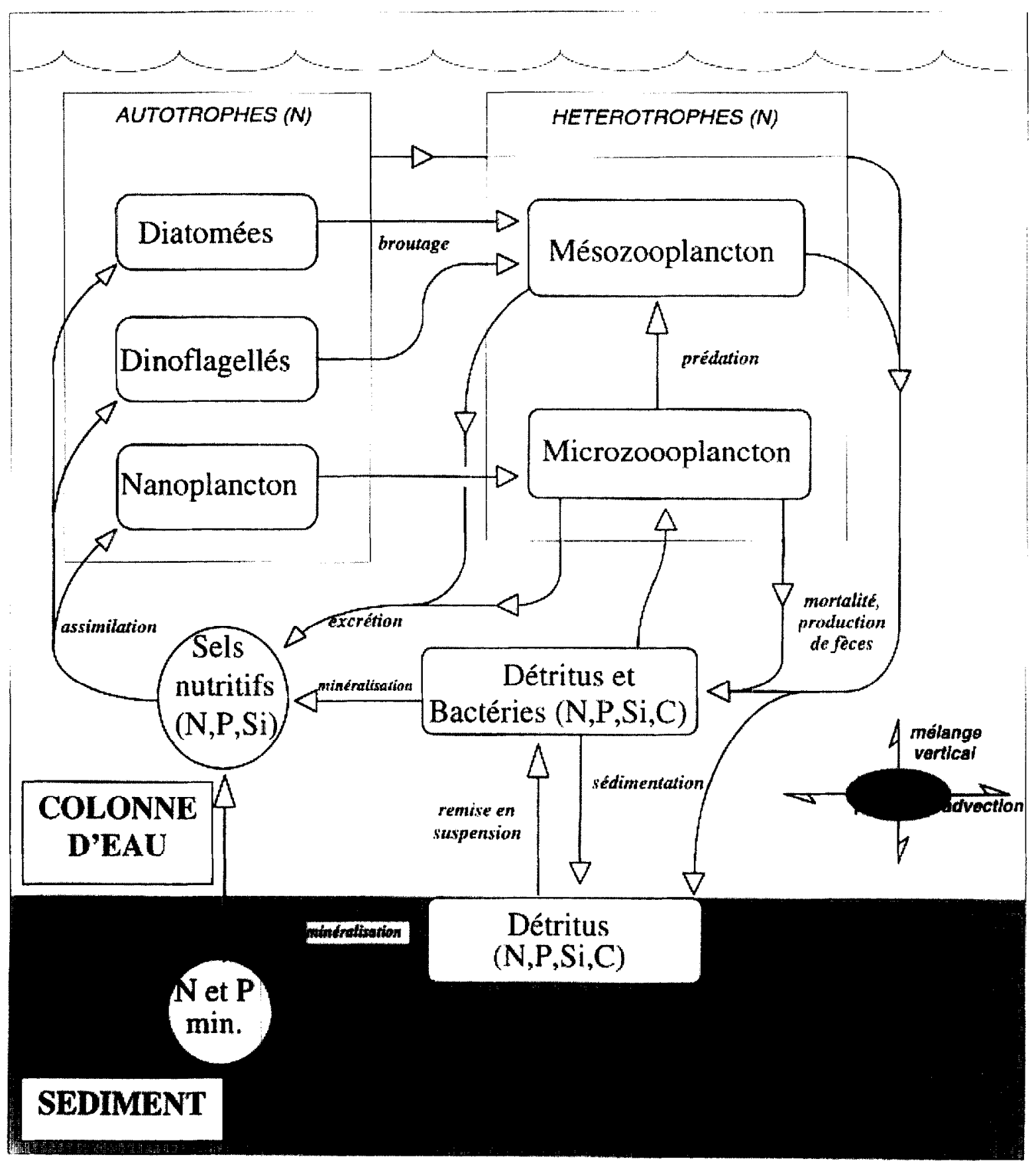

Figure 1. Schéma conceptuel du modèle biologique.

Figure 1. Conceptual scheme of the biological sub-model. 
Tableau I. Liste des équations appliquées aux nouvelles variables du modèle biologique.

Table I. List of the equations applied to the new variables of the biological submodel.

- Evolution de l'azote du nanoplancton (Nano en $\left.\mu \mathrm{mol} \mathrm{L} \mathrm{L}^{-1}\right)$ :

$$
\frac{\mathrm{dNano}}{\mathrm{dt}}=\left(\mu_{\text {Nano }}-\text { mort }_{\text {Nano }}\right) \text { Nano }- \text { ration }_{\text {Nanu }} \text { Micro }
$$

Micro : concentration en azote du microzooplancton $\left(\mu \mathrm{mol} \mathrm{L}^{-1}\right)$ :

ration $_{\text {Nano }}$ : taux de broutage du nanoplancton par le microzooplancton (en $j^{-1}$ )

* Croissance $\left(\mathrm{j}^{-1}\right): \mu_{\mathrm{Nano}}=\mu_{\max } \cdot \mathrm{f}_{\theta} \cdot \min \left(\mathbf{f}_{\mathrm{L}}, \mathbf{f}_{\mathrm{N}}, \mathbf{f}_{\mathrm{P}}\right)$

- $\mathrm{f}_{\theta}$ : facteur multiplicatif lié à la température $\theta: \quad \mathrm{f}_{\theta}=\mathrm{c}^{\left(\mathrm{k}_{\theta} \theta\right)}$

$$
\text { - Action de la lumière : } f_{L}=\iint\left(\frac{I_{z, t}}{I_{\text {sat }}}\right) e^{\left(1-\frac{I_{2, t}}{I_{s a t}}\right)} \cdot d z . d t \text { (formule de Steele) }
$$

Intensité lumineuse à la profondeur $z: I_{z, 1}=I_{0, r} \cdot e^{-k_{L} \cdot z}$

$I_{0, t}$ : intensité lumineuse en surface $\left(W \cdot \mathrm{m}^{-2}\right)$

$\mathrm{k}_{\mathrm{L}}$ : coefficient d'extinction $\left(\mathrm{en}^{-1}\right)$ :

$k_{L}=k_{N C}+k_{1} \cdot(\text { Diat }+N a n o)^{\frac{2}{3}}+k_{2} \cdot($ Diat $+N a n o)+k_{3} \cdot$ DinoCell

Diat : concentration en azote des diatomées $\left(\mu \mathrm{mol} \mathrm{L} \mathrm{L}^{-1}\right)$ :

DinoCell : nombre de cellules de dinoflagellés (cell $\mathrm{L}^{-1}$ ) :

- Action des sels nutritifs azotés ou phosphorés (formule de Michaelis-Menten) :

$$
\begin{aligned}
& f_{\text {Nut }}=\frac{[\mathrm{Nut}]}{[\mathrm{Nut}]+\mathrm{k}_{\mathrm{Nut}}} \text { avec Nut }=\mathrm{N} \text { ou } \mathrm{P} \\
& \mathrm{k}_{\mathrm{Nut}} \text { : coefficient de demi-saturation }
\end{aligned}
$$

* Mortalité $\left(\mathrm{j}^{-1}\right)$ : mort Nano $=\mathrm{m}_{0 \text { Nano }} . \mathrm{f}_{\theta}$

- Evolution du mésozooplancton (Méso en $\mu \mathrm{mol} \mathrm{L} \mathrm{L}^{-1}$ ) :

$$
\begin{aligned}
& \frac{\mathrm{dMéso}}{\mathrm{dt}}=\left(\mathrm{a}_{\text {Méso }}, \text { ration }_{\text {Méso }}-\mathrm{exc}_{\text {Méso }}-\text { mort }_{\text {Méso }}\right) \text { Méso } \\
& \text { * Broutage : ration } \text { Méso }=\mathrm{r}_{\max \text { Méso }} \cdot \mathrm{f}_{\theta} \cdot\left(1-\mathrm{e}^{-\mathrm{k}_{\mathrm{w}} \cdot \max \left(0, \text { Capt }_{\text {Mtoo }}-\mathrm{S}_{\text {Nutr }}\right)}\right) \\
& \text { Capt }_{\mathrm{Mes}} \text { : ration disponible pour le mésozooplancton } \\
& \mathrm{Capt}_{\text {Méso }}=\mathrm{Capt}_{\text {Diat }}+\mathrm{Capt}_{\text {Dino }}+\mathrm{Capt}_{\text {Micro }} \\
& \text { Capt }_{\text {Diat }}=\text { Diat } \\
& \text { Capt }_{\text {Dino }}=0,1 \text {. Dino } \\
& \text { Capt }_{\text {Micro }}=0,7 . \text { Micro }
\end{aligned}
$$

* Assimilation de l'azote : $\mathrm{a}_{\mathrm{NMéso}}=0,3,\left(3-0,67 \cdot \frac{\text { ration }_{\text {Méso }}}{\mathrm{r}_{\max M \text { Méso }_{0}} \mathrm{f}_{\theta}}\right)$

* Assimilation du phosphore : $a_{\text {PMeso }}=a_{\text {NMéso }} \cdot\left(\frac{\left(\frac{\mathrm{P}}{\mathrm{N}}\right)_{\text {Méso }}}{\left(\frac{\mathrm{P}}{\mathrm{N}}\right)_{\text {rationMéso }}}\right)$

* Excrétion : $\mathrm{e}_{\text {Méso }}=\mathrm{e}_{\max \text { Méso }}\left(\frac{\text { ration }_{\text {Mésa }}}{\mathrm{r}_{\max \text { Meso }} \cdot \mathrm{f}_{\theta}}\right)$

* Mortalité : $\mathrm{m}_{\text {Méso }}=\mathrm{m}_{0 \text { Méso }} \cdot \mathrm{f}_{\theta} \cdot$ Méso ${ }^{2}$

- Ingestion du microzooplancton :

$$
\begin{aligned}
& \text { ration }_{\text {Micro }}=\mathrm{r}_{\text {max Micro }} \cdot \mathrm{f}_{\theta} \cdot \frac{\text { Capt }_{\text {Micro }}}{\text { Capt }_{\text {Micro }}+\mathrm{k}_{\text {Capt }}} \\
& \text { Capt }_{\text {Micro }}=\text { Capt }_{\text {Nano }}+\text { Capt }_{\text {Det }} \\
& \text { Capt }_{\text {Nano }}=\text { Nano } \\
& \text { Capt }_{\text {Det }}=0,8 . \text { Det }
\end{aligned}
$$

Det : concentration en azote du compartiment détritique et bactérien $\left(\mu \mathrm{mol} \mathrm{L}^{-1}\right)$ : 
gouvernent la conception de ce modèle. Les tableaux I et $I I$ fournissent les équations du modèle pour les variables nouvellement introduites, ainsi que les paramètres correspondants. L'ensemble des équations du modèle est regroupé en annexe.

Une variable nanoplancton, dont la croissance est limitée par le phosphore et l'azote, a été ajoutée au compartiment des autotrophes (éq. I). De même que pour les autres catégories phytoplanctoniques, le taux de croissance des nanoflagellés dépend de la limitation par les éléments nutritifs ( $\mathrm{N}$ et $\mathrm{P}$ ) et par l'énergie lumineuse utilisable dans la photosynthèse (éq. 2 à 7). Suivant la loi de Liebig, le minimum des fonctions limitantes a été considéré. Il est à noter que le coefficient d'extinction lumineuse est la somme d'un coefficient d'origine non chlorophyllienne $\left(\mathrm{k}_{\mathrm{NC}}\right)$ et des influences respectives des concentrations en azote des diatomées et des nanoflagellés suivant une formule de Riley [32], et du nombre de cellules de dinoflagellés. La mortalité des cellules nanoplanctoniques est un processus de premier ordre et dépend, comme pour les autres organismes autotrophes, d'un taux de mortalité basal et de l'action de la température (éq. 8). Les taux de croissance et de mortalité des nanoflagellés ont été considérés comme plus forts que ceux des autres classes de phytoplancton, traduisant ainsi un renouvellement des cellules plus important.

Pour les hétérotrophes, deux catégories zooplanctoniques sont prises en compte.

(1) Le microzooplancton, qui réunit des protozoaires et des larves de copépodes, de tailles inférieures à $200 \mu \mathrm{m}$, ingère des cellules phytoplanctoniques de petites tailles, du matériel détritique et des bactéries. Ces deux dernières catégories sont regroupées dans le même compartiment pour tenir compte des bactéries liées aux particules détritiques, et parce que nous nous intéressons plus au recyclage des éléments par les bactéries qu'à la dynamique propre de ce compartiment.

(2) Le mésozooplancton qui broute préférentiellement des diatomées et des dinoflagellés, mais ingère également des microhétérotrophes. L'évolution temporelle de cette dernière variable est formalisée par l'équation 9. Le brou-

Tableau II. Liste des paramètres appliqués aux nouvelles variables du modèle biologique.

Table II. List of the parameters applied to the new variables of the biological sub-model.

\begin{tabular}{|c|c|c|}
\hline \multicolumn{3}{|c|}{ Nanoplancton } \\
\hline$\mu_{\text {maxnano }}$ & Taux de croissance maximal à $0^{\circ} \mathrm{C}$ & $1 j^{-1}$ \\
\hline $\mathrm{I}_{\text {satdino }}$ & Intensité lumineuse optimale & $70 \mathrm{~W} \mathrm{~m}^{-2}$ \\
\hline $\mathrm{k}_{\text {kn nario }}$ & Constante de demi-saturation pour l'azote minéral & $1 \mu \mathrm{mol} \mathrm{L} \mathrm{L}^{-1}$ \\
\hline $\mathrm{k}_{\text {Pnann }}$ & Constante de demi-saturation pour le phosphore minéral & 0,1 j $\mathrm{mol} \mathrm{I}^{-1}$ \\
\hline $\mathrm{m}_{\text {nane }}$ & Taux de mortalité à $0^{\circ} \mathrm{C}$ & $0,1 j^{-1}$ \\
\hline \multicolumn{3}{|c|}{ Mésozooplancton } \\
\hline$r_{C / N Z(x)}$ & Rapport $\mathrm{C} / \mathrm{N}$ & $4,67 \mathrm{~mol} \mathrm{~mol}^{-1}$ \\
\hline$r_{\text {maxzous }}$ & Ration maximale ingérée à $0^{\circ} \mathrm{C}$ & $0.3 \mathrm{j}^{-1}$ \\
\hline $\mathrm{k}_{\mathrm{Iv}}$ & Coefficient d'Ivlev & $0,081 \mu \mathrm{mol} \mathrm{L}-1$ \\
\hline$S_{\text {Nutr }}$ & Seuil d'échappement à la prédation & $0,5 \mu \mathrm{mol} \mathrm{L}-1$ \\
\hline $\operatorname{cxcr}_{\max r o 0}$ & Taux d'excrétion maximal à $0^{\circ} \mathrm{C}$ & $0,03 \mathrm{j}^{\prime}$ \\
\hline $\mathrm{m}_{\text {zoo }}$ & Taux de mortalité à $0^{\circ} \mathrm{C}$ & $0,1\left(1 \mu \mathrm{mol} \mathrm{L}{ }^{-1}\right) \cdot j^{-1}$ \\
\hline \multicolumn{3}{|c|}{ Microzouplancton } \\
\hline$r_{C / N \text { micro }}$ & Rapport C/N & $5,5 \mathrm{~mol} \mathrm{~mol}^{-1}$ \\
\hline 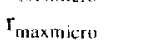 & Ration maximale ingérée à $0{ }^{\circ} \mathrm{C}$ & $0,3 \mathrm{j}^{-1}$ \\
\hline$k_{\text {Nut }}$ & Coefficient de demi-saturation & $1 \mu \mathrm{mol} \mathrm{L} \mathrm{L}^{-1}$ \\
\hline excr $_{\text {maxmicro }}$ & Taux d'excrétion maximal à $0^{\circ} \mathrm{C}$ & $0,1 \mathrm{j}^{-1}$ \\
\hline $\mathrm{m}_{\text {micro }}$ & Taux de mortalité à $0^{\circ} \mathrm{C}$ & $0,015\left(1 \mu \mathrm{mol}^{-1}\right) \mathrm{j}^{-1}$ \\
\hline \multicolumn{3}{|c|}{ Matière organique } \\
\hline $\mathrm{v}_{\mathrm{SMO}}$ & Taux de sédimentation de la matière organique & $1 \mathrm{mj}^{-1}$ \\
\hline$r_{\operatorname{mink}}$ & Taux de reminéralisation de l'azote détritique à $0{ }^{\circ} \mathrm{C}$ & $0,04 \mathrm{j}^{-1}$ \\
\hline$r_{\min P}$ & Taux de reminéralisation du phosphore détritique à $0^{\circ} \mathrm{C}$ & $0,1 j^{-1}$ \\
\hline$r_{\min C}$ & Taux de reminéralisation du carbone détritique à $0^{\circ} \mathrm{C}$ & $0,02 j^{-1}$ \\
\hline$r_{\text {diss }}$ & Taux de dissolution de la silice particulaire à $0^{\circ} \mathrm{C}$ & $0,03 j^{-1}$ \\
\hline
\end{tabular}


tage et la prédation par le mésozooplancton sont simulés par une fonction d'Ivlev (éq. 10). Dans l'ordre décroissant de préférence, le mésozooplancton ingère des diatomées, du microzooplancton et des dinoflagellés. La fraction de biomasse des diatomées broutée par le mésozooplancton s'exprime alors de la façon suivante: ration $_{\text {Méso }} \cdot \frac{\text { Cap̣t }_{\text {Diat }}}{\text { Capt }_{\text {Méso }}}$, et de manière symétrique pour les dinoflagellés et le microzooplancton. D’après Hofmann et Ambler [10], le taux d'assimilation des composés azotés par le zooplancton dépend de la ration ingérée par ces organismes. Ce taux d'assimilation est d'autant plus élevé que la ration est faible, et varie de 0,7 à 0,9 (éq. $/ 1)$. Les taux d'assimilation du phosphore et du carbone sont calculés de façon à respecter les rapports stœchiométriques constants au sein du zooplancton en fonction de ceux de la nourriture et du taux d'assimilation de l'azote. À titre d'exemple, le taux d'assimilation du phosphore est fourni par l'équation 12. Le taux d'assimilation de l'azote peut être ajusté pour que celui du phosphore ne dépasse pas la valeur de 1. Le taux d'excrétion de l'azote par le mésozooplancton est également considéré comme dépendant de la ration ingérée par les organismes (éq. 13). D’après Steele et Henderson [35], le taux de mortalité du mésozooplancton est un processus du second ordre, lié à la concentration environnante de ces individus (éq. 14).

Contrairement à celle du mésozonplancton, la nutrition du microzooplancton est formalisée par des équations de Michaelis-Menten (éq. 15). Le microzooplancton ingère préférentiellement des cellules nanoplanctoniques. Les processus d'excrétion, de mortalité, ainsi que l'assimilation des éléments sont modélisés de manière identique pour les deux catégories de zooplancton.

Le recylage des éléments s'effectue via l'excrétion des hétérotrophes, et par l'intermédiaire de la reminćralisation du matériel détritique. Ce dernier est constitué des individus morts et des pelotes fécales issues de l'égestion du zooplancton.

L'élément carbone est pris en compte par le biais de rapports stœechiométriques constants dans le phytoplancton et les héterotrophes, explicitement pour la matière organique dans l'eau et le sédiment. Le taux de recyclage du carbone est considéré comme inférieur à celui de l'azote [14]. En accord avec les taux de reminéralisation utilisés par Ross et al. [33], le laux de recyclage dans le sédiment est égal à $5 \%$ de sa valeur dans la colonne d'eau, pour les trois éléments ( $\mathrm{C}, \mathrm{N}$ et $\mathrm{P})$.
Ce modèle est utilisé pour calculer la part de la production primaire attribuable à chaque catégorie de phytoplancton [25]. Par exemple, on calcule en chaque boîte la production azotée des diatomées (en $\mu \mathrm{mol} \mathrm{m}{ }^{-2}$ ) :

$\frac{\mathrm{d} \operatorname{Prod}_{\text {Diat }}}{\mathrm{dt}}=\mu_{\text {Diat }} \cdot$ Diat.h

h représente la hauteur de la couche d'eau considérée. Les productions dans les couches de surface et de fond sont additionnées. La contribution des dinoflagellés et du nanoplancton à la production phytoplanctonique totale est calculée de manière identique. Estimée initialement en azote par le modèle, la production primaire, cumulée à l'échelle annuelle, est convertie en production carbonée à l'aide d'un rapport de Redfield [31].

Des équations similaires sont utilisées afin de simuler les flux entre les différentes variables d'état du système, pour tous les processus considérés (croissance, mortalité, broutage, prédation, égestion, excrétion; reminéralisation, sédimentation et remise en suspension). Ces flux entre compartiments sont intégrés à l'échelle annuelle et représentent la moyenne des flux calculés dans chaque boîte.

Ce modèle nous permet également de calculer en chaque boite la proportion d'azote produit par régénération par rapport à la somme des sources d'azote. L'azote de régénération est produit soit par reminéralisation dans la colonne d'eau, soit par excrétion des hétérotrophes. La quantité d'azote régênéré dans une boîte yaut :

$$
\begin{aligned}
& \frac{\mathrm{dNreg}}{\mathrm{dt}}= \\
& \left(\text { re min } \cdot \text { Det }+\operatorname{Exc}_{\text {Micro }} \cdot \text { Micro }+\operatorname{Exc}_{\text {Méso }} \cdot \text { Méso }\right) \cdot V
\end{aligned}
$$

V est le volume de la boîte considérée. À l'opposé, l'azote nouveau est constitué par le stock hivernal, l'azote reminéralisé dans le sédiment et les apports par les fleuves. Dans ce dernier cas, l'azote est considéré comme nouveau tant qu'il n'a pas été assimilé par les cellules phytoplanctoniques.

\subsubsection{Variables forçantes, conditions aux limites et données de calibration}

Les variables forçantes sont inchangées par rapport au modèle de Hoch et Ménesguen [11]. Les conditions aux. limites spatiales sont identiques pour les variables déjà présentes dans ce modèle. Pour les autres variables (nanoplancton, zooplancton et carbone organique détriti- 
que), nous considérons que les concentrations aux limites atlantiques du domaine sont équivalentes à celles simulées dans les boîtes directement influencées par ces apports. La dynamique du système n'est que peu influencée par les valeurs de ces concentrations aux limites.

Pour comparer les résultats des simulations et les mesures, nous additionnons les valeurs d'azote phytoplanctonique pour les trois compartiments (diatomées, dinoflagellés et nanoplancton), après conversion en chlorophylle $a$ à raison de $1 \mu \mathrm{g} \mathrm{L}^{-1}$ de chlorophylle pour $1 \mu \mathrm{mol} \mathrm{L}{ }^{-1}$ d'azote $[3,9]$. Le calage est effectué prioritairement en cinq boîtes :

- la boîte A, située au large de la côte anglaise en zone temporairement stratifiée et qui inclue la station 2, échantillonnée dans le cadre du PNOC-Chantier Manche, et pour laquelle nous utilisons également des données de la littérature [12],

- la boîte B, au nord de la baie de Morlaix, comprenant la station 1 ,

- la boîte C, dans la baie du mont Saint-Michel, avec des données de Le Hir et al. [15],

- la boîte $\mathrm{D}$, dans l'estuaire de la Seine, où les résultats des simulations sont comparés à des données du RNO (Réseau national d'observation de la qualité du milieu marin), réseau de surveillance français,

- la boîte E, dans le détroit du Pas-de-Calais, incluant la station 6, au travers duquel Bentley [5] a également effectué de nombreuses mesures en 1980.

À l'exception de la station 2, échantillonnée à l'occasion de la campagne printanière CPB 1 du PNOC [29], les stations ont fait l'objet d'un suivi saisonnier dans le cadre du programme.

\section{RÉSULTATS DES SIMULATIONS}

La dynamique de l'écosystème est décrite à partir de la comparaison entre les résultats du modèle et les mesures. Nous exploitons ensuite ce modèle de façon à dégager une vision synoptique de la production primaire à l'échelle de la Manche.

\subsection{Comparaison des résultats du modèle avec des données de terrain (figure 2)}

L'apparition du bloom printanier est principalement liéc à la hauteur de la couche de surface. Lorsque la colonne d'eau est stratifiée, la thermocline ou l'halocline isolent une couche de surface peu épaisse, où l'énergie lumineuse favorise le développement phytoplanctonique. Ainsi, (figure 2), le bloom phytoplanctonique apparaît plus tôt au large de la côte anglaise de Manche occidentale (boîte $A$ ) qu'à la côte française (boite B), en relation avec la formation de la thermocline. Après le pic printanier, la stratification stable dans la boîte $A$ engendre un appauvrissement général en chlorophylle $a$. Les teneurs phytoplanctoniques n'augmentent qu'à l'automne, au moment de la déstratification. Dans la boîte B, l'instabilité de la stratification, en raison de courants de marée plus forts, engendre une série de petits pics chlorophylliens successifs. Les zones peu profondes, comme dans la baie du mont Saint-Michel (boîte $\mathrm{C}$ ), sont aussi le siège d'une croissance phytoplanctonique précoce, mais les interactions avec le domaine benthique par le biais des processus de sédimentation et remise en suspension et de reminéralisation, n'engendrent pas un appauvrissement aussi prononcé que dans la zone stratifiée. En baie de Seine (boîte D), l'halocline engendrée par les apports d'eau douce du fleuve atténue l'effet d'une turbidité forte et favorise l'apparition d'un bloom assez précoce. La dynamique phytoplanctonique dans le Pasde-Calais (boîte E) est assez similaire à celle de la baie du mont Saint-Michel.

L'intensité du développement planctonique dépend en revanche de la disponibilité en sels nutritifs. Au débouché de la Seine (boîte D), les apports par le fleuve engendrent un bloom printanier important $\left(22 \mu \mathrm{mol} \mathrm{L} \mathrm{L}^{-1}\right.$ d'azote phytoplanctonique) et permettent de soutenir une croissance estivale continue, avec un maximum estimé aux alentours de $27 \mu \mathrm{mol} \mathrm{L}^{-1}$ au début de l'été. Les autres boîtes présentent un pic printanier maximal simulé variant entre $3,5 \mu \mathrm{mol} \mathrm{L}{ }^{-1}$ (boîte B) et un peu moins de $6 \mu \mathrm{mol} \mathrm{L} \mathrm{L}^{-1}$ d'azote phytoplanctonique dans le Pas-de-Calais.

La figure synthétise l'évolution annuelle de la chlorophylle $a$ en plusieurs points représentant l'ensemble des différents écosystèmes rencontrés en Manche. Le bloom printanier est dans l'ensemble bien reproduit par le modèle. Des différences avec les données peuvent être remarquées en période estivale, notamment dans le Pasde-Calais (boîte E). Ceci peut être imputé à l'influence du benthos, non détaillée dans le modèle, ou à celle des zones côtières riches en éléments nutritifs, à cause d'un transport d'eau trop important vers le large dû à la discrétisation spatiale en boîtes. 


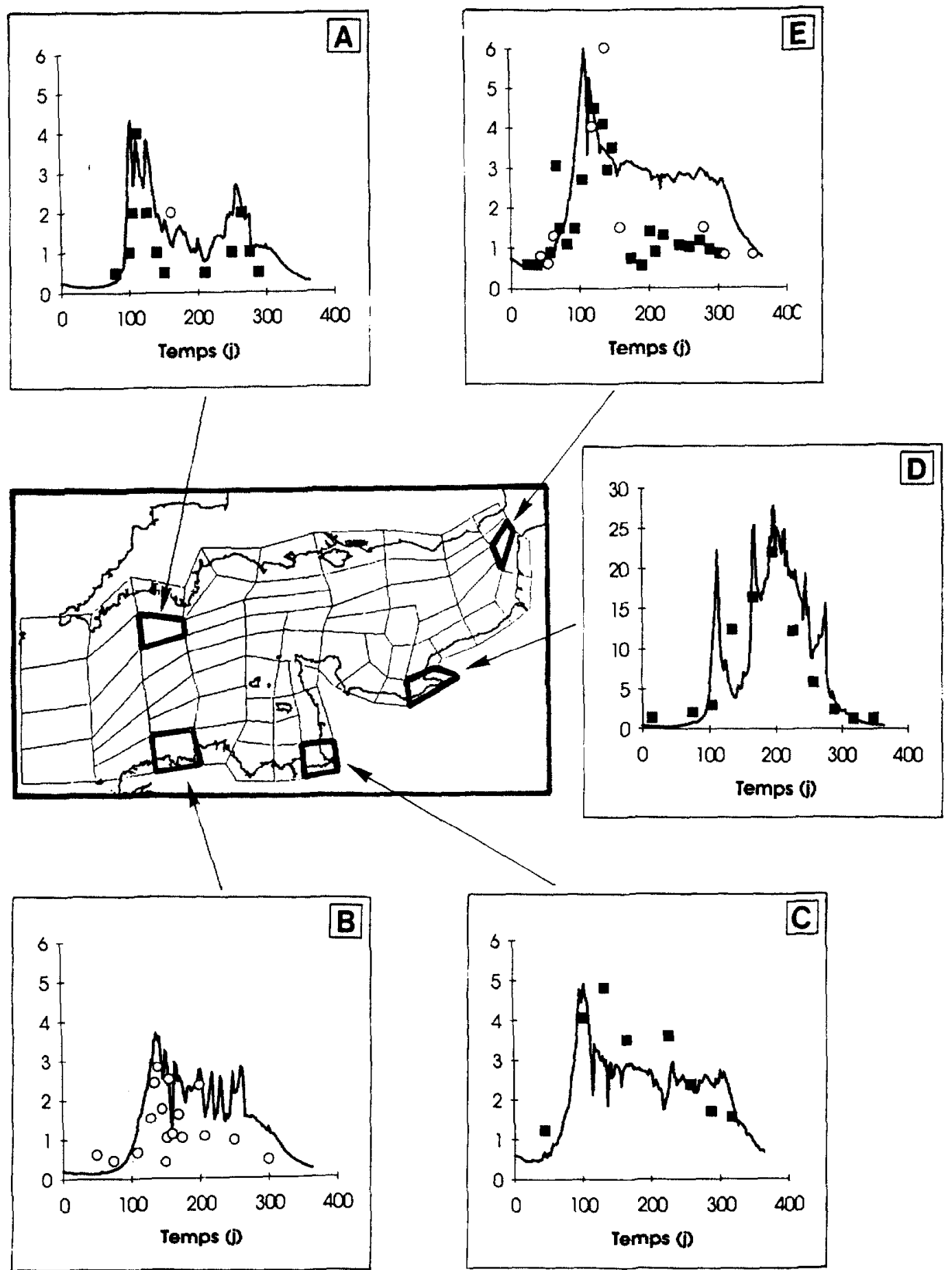

Figure 2. Comparaison entre les résultats du modèle et les mesures pour l'azote phytoplanctonique total ( $\mu$ mol $\mathrm{L}^{-1}$ ) dans la couche de surface. Les données sont issues soit de la littérature (ם), soit de mesures effectuées dans le cadre du PNOC-Chantier Manche (O). Le jour 0 correspond au $l^{\text {er }}$ janvier.

Figure 2. Comparison between model results and measurements for total phytoplanktonic nitrogen $\left(\mu \mathrm{mol} \mathrm{L}^{-1}\right)$ in the surface layer. Data are either coming from the literature $(\boldsymbol{Q})$, or measured during PNOC-Chantier Manche cruises $(O)$. Day 0 corresponds to 1 January. 
3.1.1. Distribution spatiale de la production primaire (figure 3)

Les zones côtières, malgré la plus forte disponibilité en éléments nutritifs, sont moins productives du fait de l'importance de l'extinction d'origine non chlorophyllienne, sauf dans l'estuaire de la Seine où les forts apports terrigènes engendrent des productions élevées. Dans les eaux du large, une nette distinction peut être faite entre les secteurs brassés et les régions saisonnièrement stratifiées de Manche occidentale. Dans celles-ci, la sédimentation exporte les cellules phytoplanctoniques et la matière organique détritique de la couche de surface vers celle du fond, d'où une production plus faible que dans le système homogène profond de Manche centrale et orientale. Ces deux dernières zones apparaissent comme les plus productives. Sur l'ensemble de la Manche, la production carbonée totale est estimée à un peu plus de $20 \times 10^{6} \mathrm{tan}^{-1}$.

\subsubsection{Proportion d'azote produit par régénération dans la colonne d'eau (figure 4)}

Cette carte souligne l'importance de la régénération dans les zones brassées profondes : en Manche centrale, la part d'azote produit par régénération dépasse $80 \%$. Cette situation contraste avec celle qui prévaut aux limites spatiales du modèle: au large de l'île d'Ouessant (apports aux limites atlantiques), en baie de Seine (apports continentaux) et dans les régions côtières peu profondes, telles que la baie du mont Saint-Michel (sources d'azote plus diversifiées, comprenant le flux d'origine benthique), ce pourcentage est nettement plus faible. L'ensemble des zones côtières, par leur plus forte turbidité, apparaît relativement moins productif et plus dépendant du stock hivernal de sels nutritifs. À l'échelle de la Manche, la contribution de l'azote issu de la régénération est estimée à $67 \%$.

\subsubsection{Flux d'azote au sein du système biologique}

Nous avons calculé à l'aide du modèle les flux d'azote entre les différents compartiments du système (figure 5). Ces flux n'apparaissent pas équilibrés car le schéma $n$ 'indique pas les apports aux limites ainsi que les sorties du système. La production par les diatomées représente l'essentiel de la production phytoplanctonique. La production primaire se dégrade ensuite principalement en matière organique détritique. En effet, le flux d'azote lié à

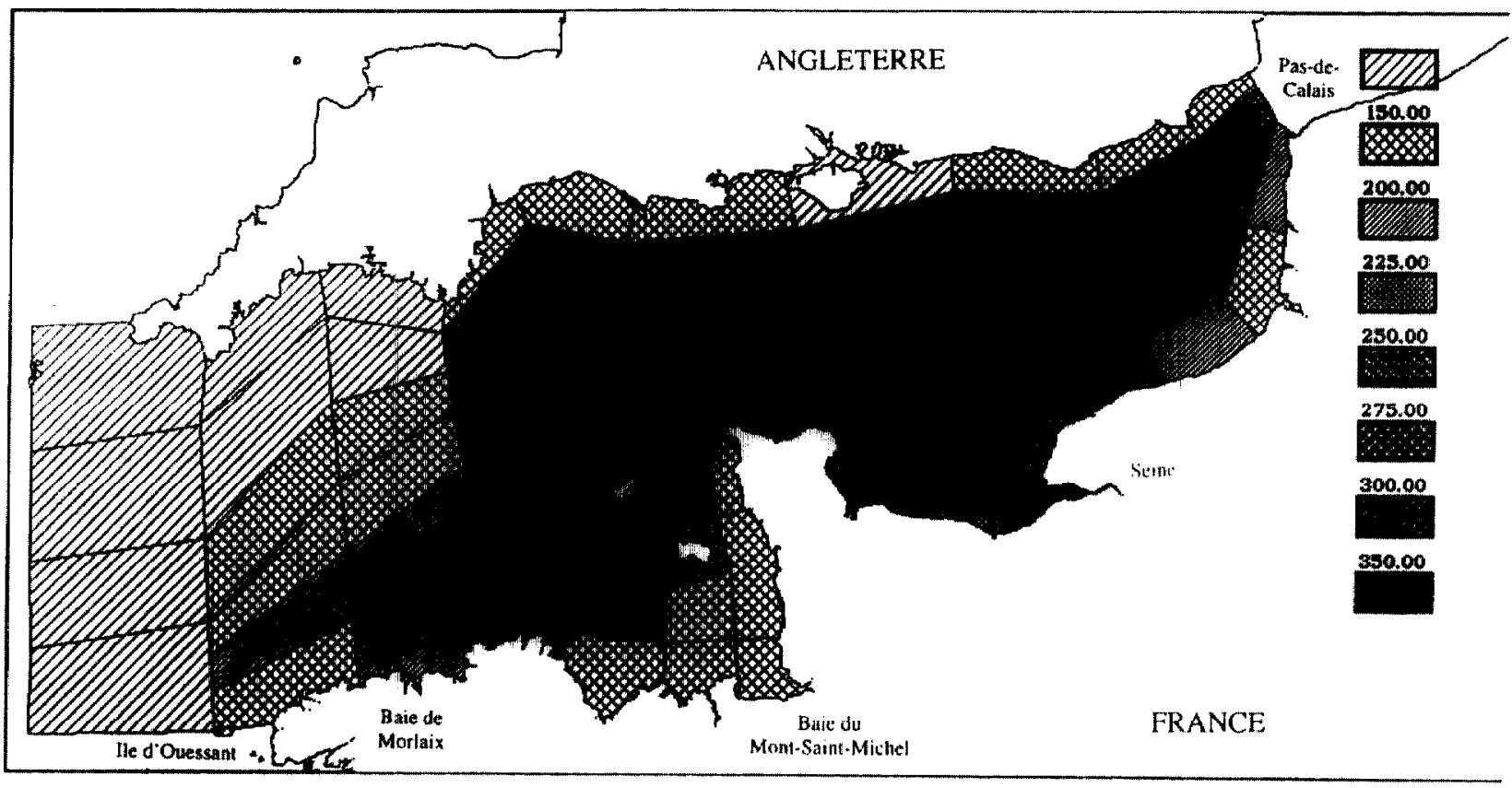

Figure 3. Carte de la production primaire carbonée cumulée sur l'année et intégrée sur la profondeur de la colonne d'eau ( $\left(\mathrm{g} \mathrm{m}^{-2}\right.$ an ${ }^{-1}$ ).

Figure 3. Map of carbon primary production calculated over one year and integrated on the depth of the water column $\left(\mathrm{g} \mathrm{m}^{-2} \mathrm{y}^{-1}\right)$. 


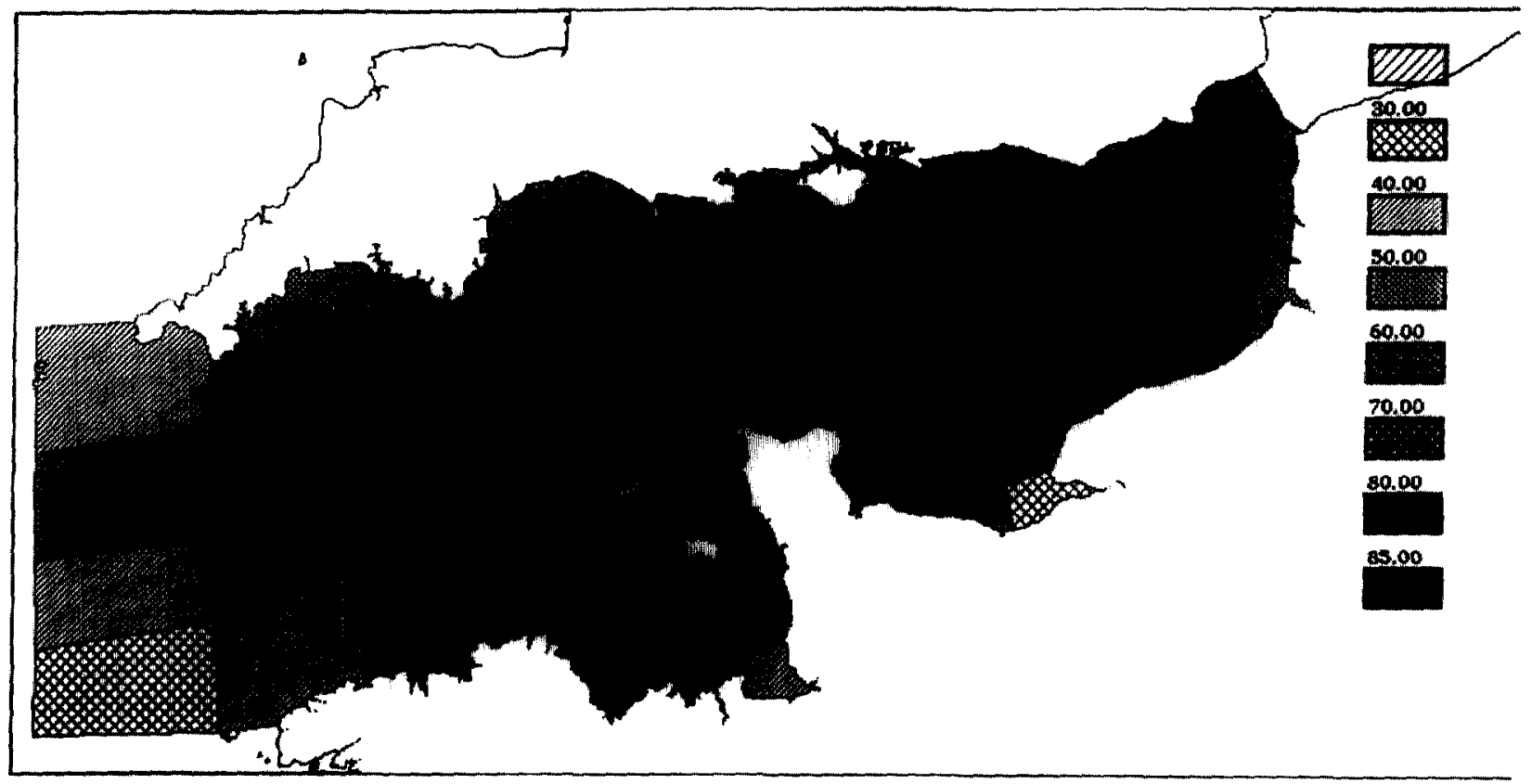

Figure 4. Carte du pourcentage simulé d'azote inorganique produit par régénération dans la colonne d'eau (\%).

Figure 4. Map of the simulated percentage of inorganic nitrogen produced by regeneration in the water column (\%).

la mortalité des diatomées, associé sur la figure à leur sédimentation et à la mortalité des autres catégories phytoplanctoniques, représente $20,73 \mathrm{~g} \mathrm{~m}^{-2}$, soit près de $60 \%$ de la production de ces cellules. D'une manière générale, le rôle du mésozooplancton semble relativement limité. En revanche, d'après le modèle, le compartiment microzooplanctonique joue un rôle important, notamment du fait des interactions fortes avec le compartiment regroupant matériel détritique et bactéries. La reminéralisation par les bactéries apparaît comme la principale voie de recyclage des éléments. Les échanges avec le sédiment sont faibles mais non négligeables.

\section{CONCLUSION ET DISCUSSION}

Le modèle en boîtes simule en des temps de calcul raisonnables la structure physique horizontale et verticale en Manche, autorise à détailler le sous-modèle biologique décrivant les interactions entre les principaux compartiments du système pélagique. Ce modèle écologique fournit une vision synoptique de la dynamique phytoplanctonique à l'échelle de la Manche. Cette dynamique repose essentiellement sur deux facteurs : l'épaisseur de la couche de surface, ou la profondeur de la colonne d'eau lorsque celle-ci est totalement mélangée, et la disponibilité en éléments nutritifs. Brunet et al. [7] ont décrit en Manche orientale des différences dans la date d'apparition et la composition du bloom phytoplanctonique printanier entre deux zones situées au nord et au sud de la baie de Somme. Ils les attribuent à des profondeurs et des degrés de stabilité de la colonne d'eau distincts entre les deux régions. Ce modèle permet également l'élaboration d'une carte de la production primaire annuelle, influencée par la structure physique verticale ainsi que par la turbidité en milieu côtier. La prise en compte du nanoplancton paraît nécessaire car, si elle ne modifie que peu l'évolution des concentrations phytoplanctoniques, elle permet d'augmenter les valeurs des productions simulées. Celles-ci, sous-estimées par le précédent modèle [25] sont maintenant comparables à celles mesurées au nord de Roscoff (boîte B) par L'Helguen [19] et Maguer et al. [21] qui trouvent respectivement des productions annuelles carbonées de 360 et $250 \mathrm{~g} \mathrm{~m}^{-2}$. De même, l'intégration à l'échelle annuelle des mesures de Boalch [6] amène à des productions de l'ordre de $150 \mathrm{~g} \mathrm{~m}^{-2}$ au large de la côte anglaise de Manche occidentale (boîte A), tandis que Quisthoudt [30] trouve une production annuelle de $336 \mathrm{~g} \mathrm{~m}^{-2}$ dans une bande côtière du Pas-de-Calais. Si des sous-estimations des productions 


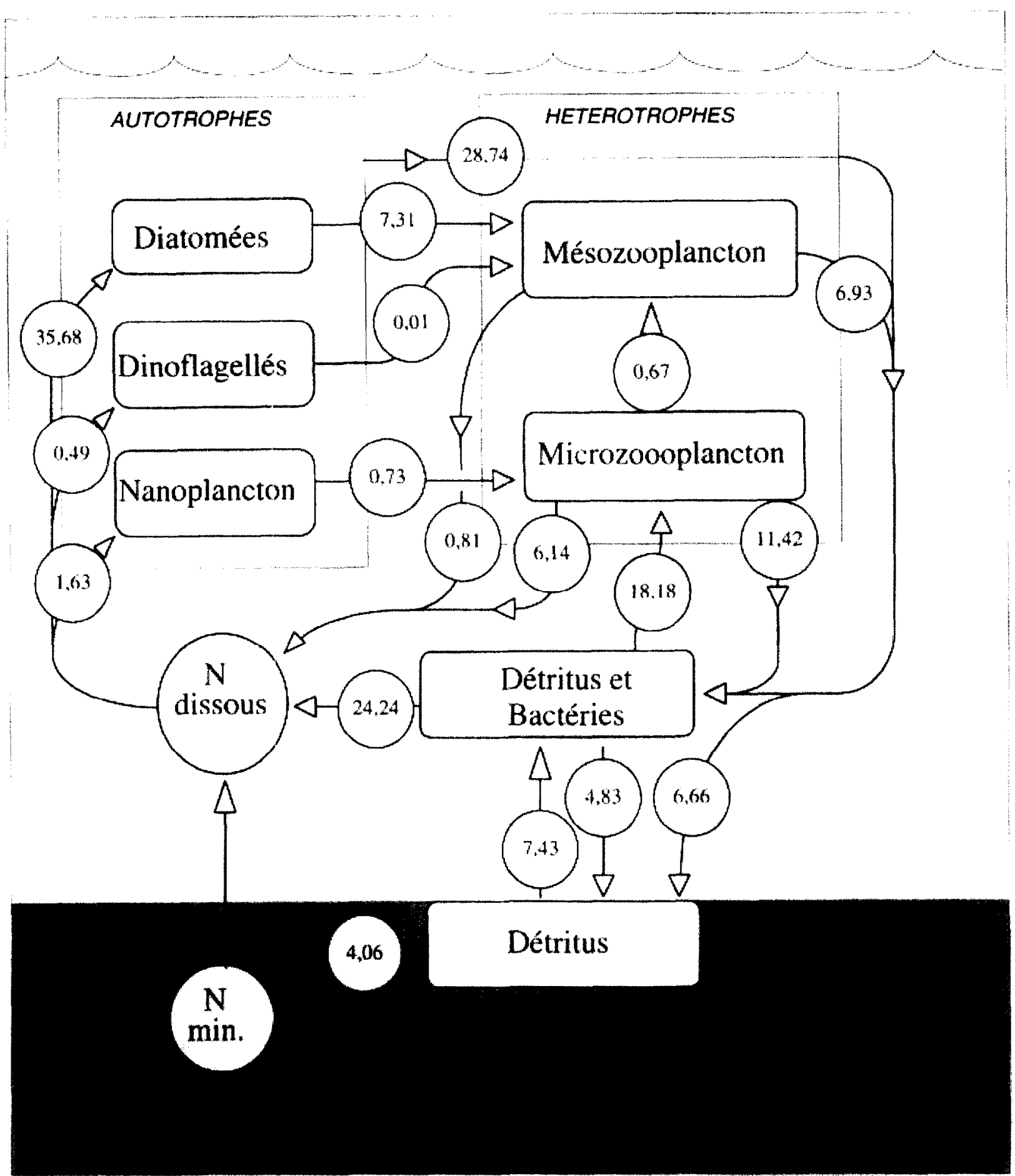

Figure 5. Flux annuels d'azote $\left(\mathrm{g} \mathrm{m}^{-2}\right)$ estimés entre les compartiments biologiques du système : moyennes des flux simulés dans chaque boîte.

Figure 5. Annual nitrogen fluxes $\left(\mathrm{g} \mathrm{m}^{-2}\right)$ estimated between the different compartments of the system: average of simulated fluxes in each box. 
calculées subsistent, elles sont attribuables, soit à une stratification simulée trop prononcée, soit à une turbidité estimée trop forte, soil enfin à des flux benthiques d'éléments nutritifs trop faibles.

Cette étude a mis en évidence le rôle central joué par la régénération des éléments, notamment de l'azote, au sein de la colonne d'eau. La contribution de l'azote inorganique produit par ce processus représente environ les 2/3 des sources totales d'azote mineral. Cette prédominance a été mise en évidence par L'Helguen et al. [20] et Maguer et al. [22]. Ces derniers auteurs montrent que dans l'ensemble du système brassé de la Manche, la régénération de l'ammonium par les microhétérotrophes satisfait en moyenne $80 \%$ des besoins du phytoplancton, en accord avec les valeurs simulées dans les zones peu soumises aux apports externes. Les résultats expérimentaux ainsi que la simulation amènent à conclure que la production primaire à l'échelle de la Manche est essentiellement d'origine régénérée.

La reminéralisation bactérienne domine dans notre modèle pour ce qui concerne le recyclage des éléments. D'après Le Corre et al. [16], les flux d'ammonium liés à la reminéralisation concernent l'ensemble de la boucle microbienne, dans le sens : matière organique $\rightarrow$ bactéries $\rightarrow$ nanoflagellés $\rightarrow$ ciliés. Ainsi, dans une zone brassée de Manche occidentale, le recyclage de l'azote est principalement dû aux microhétérotrophes qui participent pour $64 \%$ à la régénération de l'ammonium, tandis que la part attribuée aux bactéries est au maximum de $26 \%$ en automne [17]. Même si le rôle central joué par la régénération est reproduit par le modèle, sans doute faudraitil réajuster l'importance respective des différents compar- timents de la boucle microbienne. En outre, l'influence du sédiment apparaît restreinte à l'échelle de la Manche. Le Pape [18] a démontré de fortes intcrrclations entre les systèmes pélagique et benthique, via la sédimentation du matériel détritique, à l'aide d'un modèle comparable à celui présenté ici et appliqué à la rade de Brest. La profondeur de la colonne d'eau joue donc un rôle fondamental dans le couplage entre pélagos et benthos. Le modèle écologique de la rade de Brest inclut une description détaillée de l'écosystème benthique qui identifie six compartiments dans le sédiment. Afin d'améliorer la compréhension du couplage pélago-benthique, il faudrait affiner la description des processus dans le sédiment, en s'inspirant notamment du travail précité ainsi que du modèle de Chardy et Dauvin [8]. La définition des différentes strates biosédimentaires en Manche représente toutefois une tâche difficile.

La prise en compte explicite du carbone et de son assimilation dans la photosynthèse constitue un autre axe de développement possible du modèle, à l'exemple de Walsh et Dieterle [37] qui prennent en considération le $\mathrm{CO}_{2}$ dissous, les échanges de $\mathrm{CO}_{2}$ entre l'atmosphère et l'océan ainsi que la biomasse phytoplanctonique carbonée.

\section{Remerciements}

Ce travail a été effectué grâce à un financement du PNOC-Chantier Manche. La mise au point et les simulations ont été réalisées à l'aide du logiciel Élise développé à l'Ifremer par Alain Ménesguen.

\section{RÉFÉRENCES}

[1] Agoumi A., Enderlé M.J., Gras R.A., Modélisation du régime thermique en Manche, Uceanol. Acta 6 (1983) 393-406.

[2] Andersen V., Rassoulzadegan F., Modèle vertical de l'écosystème pélagique marin, Rêseau microbien et sédimentation des particules biogéniques, J. Rech. Océanogr. 16 (1991) 16-22.

[3] Antia N.J., McAllister C.D., Parsons T.R., Stephens K., Strickland J.D.H., Further measurements of primary production using a large-volume plastic sphere, Limnol. Oceanogr. 8 (1963) 166-183.

[4] Baretta-Bekker J.G., Riemann B., Baretta J.W., Koch Rasmussen E., Testing the microbial loop concept by comparing mesocosm data with results from a dynamical simulation model, Mar. Ecol. Prog. Ser. 106 (1994) 187-196.
[5] Bentley D., Contribution à l'étude hydrobiologique du détroit du Pas-de-Calais, paramètres physico-chimiques, thèse $3^{\mathrm{e}}$ cycle, univ. Sci. Techn. Lille (1984).

[6] Boalch G.T., Changes in the phytoplankton of the western English Channel in recent years, Br. Phycol. J. 22 (1987) 225235.

[7] Brunet C., Brylinski J.-M., Bodineau L., Thoumelin G., Bentley D., Hilde D., Phytoplankton dynamics during the Spring bloom in the south-eastern English Channel, Estuar. Coast. Shelf Sci. 43 (1996) 469-483.

[8] Chardy P., Dauvin J.-C., Carbon flux in a subtidal fine sand community from the western English Channel: a simulation analysis, Mar. Ecol. Prog. Ser. 81 (1992) 147-161. 
[9] Eppley R.W., Rogers J.N., McCarthy J.J., Sournia A., Light/ dark periodicity in nitrogen assimilation of the marine phytoplankters Skeletonema costatum and Coccolithus huxleyi in $\mathrm{N}$-limitant chermostat culture, J. Phycol. 7 (1971) 150-154.

[10] Hofmann E.E., Ambler J.W., Plankton dynamics on the outer southeastern U.S. continental shelf, J. Mar. Res. 46 (1988) $883-917$.

[11] Hoch T., Ménesguen A., Modelling the biogeochemical cycles of elements limiting the primary production in the Channel $(\mathrm{N}$, P, Si): Sensitivity analyses, Mar. Ecol. Prog. Ser. 146 (1997) 189-205

[12] Holligan P.M., Harbour D.S., The vertical distribution and succession of phytoplankton in the western English Channel in 1975 and 1976, J. Mar. Biol. Ass. U.K. 57 (1977) 1075-1093.

[13] Holligan P.M., Harris R.P., Newell R.C., Harbour D.S., Head R.N., Linley E.A.S., Lucas M.I., Tranter P.R.G., Weekley C.M., Vertical distribution and partitioning of organic carbon in mixcd, frontal and stratified watcrs of the English Channel, Mar. Ecol. Prog. Ser. 14 (1984) 111-127.

[14] Knauer G.A., Ayers A.V., Changes in carbon, nitrogen, adenosine triphosphate and chlorophyll $a$ in decomposing Thalassia testudinum leaves, Limnol. Oceanogr. 22 (1977) 408-414.

[15] Le Hir P., Bassoulet P., Erard E., Blanchard M., Hamon D., Jégou A.-M., Iriec (Institut de recherche en informatique et économie). Étude régionale intégrée du golfe Normand-Breton, 2. Milieu pélagique, rapp. Ifremer/Dero-E] 86.27, Ifremer, Brest (1986)

[16] Le Corre P., L'Helguen S., Wafar M., Nitrogen source for uptake by Gyrodinium cf. aureolum in a tidal front, Limnol. Oceanogr. 38 (1993) 446-451.

[17] Le Corre P., Wafar M., L'Helguen S., Maguer J.-F., Ammonium assimilation and regeneration by size-fractionated plankton in permanently well-mixed temperate waters, J. Plankt. Res. 18 (1996) 355-370.

[18] Le Pape $\mathrm{O}$., Modélisation des cycles biogéochimiques des éléments limitant la production phytoplanctonique en rade de Brest, thèse Ensa Rennes, mention halicutique (1996) 191 p.

[19] L'Helguen S., Absorption et régénération de l'azote dans les écosystèmes pélagiques du plateau continental de la Manche occidentale, relations avec le régime de mélange vertical des masses d'eau : cas du front thermique d'Ouessant. thèse doct. chimie appliquée : chimie marine, univ. Bretagne occidentale, Brest (1991) 212 p.

[20] L'Helguen S., Madec C., Le Corre P., Nutrition azotée du phytoplancton dans les eaux brassées de Manche occidentale, Oceanol. Acta 16 (1993) 653-660.

[21] Maguer J.-F., Le Corre P., L'Helguen S., Sources d'azote pour le phytoplancton dans le système brassé peu profond de la Manche occidentale, Importance de la production régénérée : bilan annuel, J. Rech. Océanogr. 21 (1996) 103-108.

[22] Maguer J.-F., L'Helguen S., Madec C., Le Corre P., Absorption et régénération de l'azote dans le système brassé de la
Manche: production nouvelle et régénérée, Oceanol. Acta 21 (6) (1998) 861-872.

[23] Mann K.H., Ecology of coastal waters, A system approach, Studies in Ecology, Univ. California Press. Los Angeles, 1982, $322 \mathrm{p}$.

[24] Ménesguen A., Élise an interactive software for modelling complex ayuatic ecosystems, in: Arcilla A.S., Pastor M., Zienkiewicz O.C., Schrefler B.A. (éd.), Computer Modelling in Ocean Engineering 91, Balkema, Rotterdam (1991) 87-94.

[25] Ménesguen A., Hoch T.. Modelling the biogeochemical cycles of elements limiting the primary production in the Channel $(\mathrm{N}$. $\mathrm{P}, \mathrm{Si})$ : I The role of thermohaline stratification, Mar. Ecol Prog. Ser. 146 (1997) 173-188.

[26] Moloney C.L., Field J.G., The size-based dynamics of plankton food webs. I. A simulation model of carbon and nitrogen flows, J. Plankt. Res. 13 (1991) 1003-1038.

[27] Niiler P.P., Kraus E.B., One-dimensional models of the upper ocean, in: Kraus E.B. (Ed.), Modelling and prediction of the upper layers of the ocean, Proceedings of a NATO advanced Study Institute, Pergamon Press, (1977) 145-172.

[28] Painting S.J., Moloney C.L., Lucas M.I., Simulation and field measurements of phytoplankton-bacteria-zooplankton interactions in the southem Benguela upwelling region, Mar. Ecol. Prog. Ser 100 (1993) 55-69.

[29] Programme national d'océanographie côtière, chantier Manche, bilan synthétique des actions de recherche en cours et des résultats acquis au 30 septembre 1995 (1995) 51 p.

[30] Quisthoudt C., Production primaire phytoplanctonique dans le détroit du Pas-de-Calais (France): variations spatiales et annuelles au large du Cap Gris-Nez, C.R. Acad. Sci. Paris. 10 (3) (1987) 245-250.

[31] Redfield A.C., On the proportions of organic derivatives in sea water and their relation to the composition, in: James Johnstone Memorial Volume, univ. Liverpool (1934) 176-192.

[32] Riley G.A., Transparency-chlorophyll relations, Limnol. Oceanogr. 20 (1975) 150-152.

[33] Ross A.H., Gurney W.S.C., Heath M.R., Hay S.J., Henderson E.W., A strategic simulation model of a fjord ecosystem, Limnol. Oceanogr. 38 (1993) 128-153.

[34] Salomon J.-C., Breton M., An atlas of long term currents in the Channel, Oceanol. Acta 16 (1993) 439-448.

[35] Steele J.H., Henderson E.W., The role of predation in plankton models, J. Plankt. Res. 14 (1992) 157-172.

[36] Vézina A.F., Platt T., Food web dynamics in the ocean. I. Bestestimates of flow networks using inverse methods, Mar. Ecol. Prog. Ser. 42 (1988) 269-287.

[37] Walsh J.J., Dieterle D.A. CO, cycling in the ocean. I. A numerical analysis of the southeastern Bering Sea with applications to the Chukchi Sea and the northern Gulf of Mexico, Prog. Oceanogr. 34 (1994) 335-392. 


\section{ANNEXE : Équations différentielles du sous-modèle biologique}

$\mathrm{N}_{\text {min }} \quad:$ azote minéral $\left(\mu \mathrm{mol} \mathrm{L} \mathrm{L}^{-1}\right)$

$\mathrm{Si}_{\text {diss }} \quad:$ silice dissoute $\left(\mu \mathrm{mol} \mathrm{L} \mathrm{L}^{-1}\right)$

$\mathrm{P}_{\min } \quad$ : phosphore minéral $\left(\mu \mathrm{mol} \mathrm{L} \mathrm{L}^{-1}\right)$

Diat : azote des diatomées $\left(\mu \mathrm{mol} \mathrm{L} \mathrm{L}^{-1}\right.$ )

DinoCell : abondance des dinoflagellés (cell $\mathrm{L}^{-1}$ )

DinoN : azote des dinoflagellés $\left(\mathrm{pmol} \mathrm{L}^{-1}\right)$

DinoP : phosphore des dinoflagellés $\left(\mathrm{pmol} \mathrm{L}^{-1}\right.$ )

Nano : azote du nanoplancton $\left(\mu \mathrm{mol} \mathrm{L} \mathrm{L}^{-1}\right)$

Méso : azote du mésozooplancton $\left(\mu \mathrm{mol} \mathrm{L}^{-1}\right)$

Micro : azote du microzooplancton $\left(\mu \mathrm{mol} \mathrm{L}{ }^{-1}\right)$

$\mathrm{N}_{\mathrm{det}} \quad$ : azote détritique dans l'eau $\left(\mu \mathrm{mol} \mathrm{L} \mathrm{L}^{-1}\right)$

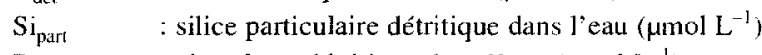

$\mathrm{P}_{\text {det }} \quad$ : phosphore détritique dans l'eau $\left(\mu \mathrm{mol} \mathrm{L} \mathrm{L}^{-1}\right)$

$\mathrm{C}_{\text {det }} \quad$ : carbone détritique dans l'eau $\left(\mu \mathrm{mol} \mathrm{L} \mathrm{L}^{-1}\right)$

$\mathrm{N}_{\text {ben }} \quad$ : azote organique benthique $\left(\mu \mathrm{mol} \mathrm{\textrm {m } ^ { - 2 }}\right)$

$\mathrm{Si}_{\text {ben }} \quad:$ silice benthique $\left(\mu \mathrm{mol} \mathrm{m} \mathrm{m}^{-2}\right)$

$\mathrm{P}_{\text {ben }} \quad:$ phosphore organique benthique $\left(\mu \mathrm{mol} \mathrm{\textrm {m } ^ { - 2 }}\right)$

$\mathrm{C}_{\text {ben }} \quad$ : carbone organique benthique $\left(\mu \mathrm{mol} \mathrm{m} \mathrm{m}^{-2}\right)$

$\frac{d N_{\text {min }}}{d t}=r_{\min N} \cdot N_{d e t}+r_{\text {minNben }} \cdot N_{\text {ben }}+\operatorname{excr}_{\text {Méso }} \cdot$ Méso $+\operatorname{excr}_{\text {Micro }} \cdot$ Micro $-\mu_{\text {Diat }} \cdot$ Diat -assimN $\cdot$ DinoCell $-\mu_{N a n o} \cdot N a n o$

$\frac{d S i_{d i s s}}{d t}=r_{d i s s} \cdot S i_{\text {part }}-\mu_{\text {Diat }} \cdot$ Diat

$\frac{d P_{\min }}{d t}=r_{\min P} \cdot P_{d e t}+r_{\min P b e n} \cdot P_{b e n}+\operatorname{excr}_{\text {Méso }} \cdot$ Méso + excr ${ }_{\text {Micro }} \cdot$ Micro $-\mu_{\text {Diat }} \cdot$ Diat - assim $P \cdot$ DinoCell- $\mu_{\text {Nano }} \cdot N a n o$

$\frac{\mathrm{dDiat}}{\mathrm{dt}}=\left(\mu_{\text {Diat }}-\right.$ mort $_{\text {Dial }}-$ sédim $\left._{\text {Diat }}\right) \cdot$ Diat - ration Méso ${ }_{\text {Diat }} \cdot$ Méso

$\frac{\mathrm{dDinoCell}}{\mathrm{dt}}=\left(\mu_{\text {Dino }}-\right.$ mort $\left._{\text {Dino }}\right) \cdot$ DinoCell - rationMéso $\operatorname{Dino}_{0} \cdot\left(\frac{\text { DinoCell }}{\operatorname{DinoN}}\right) \cdot$ Méso

$\frac{\mathrm{dDinoN}}{\mathrm{dt}}=\operatorname{assimN} \cdot$ DinoCell - mort $_{\text {Dino }} \cdot \operatorname{DinoN}-$ ration Méso Dino $_{\text {Méso }}$

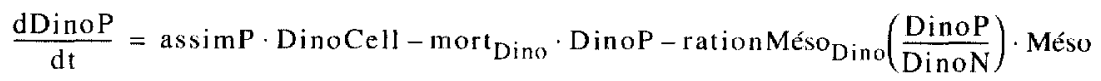

$\frac{\mathrm{dNano}}{\mathrm{dt}}=\left(\mu_{\text {Dino }}-\right.$ mort $\left._{\text {Dino }}\right) \cdot$ Nano-rationMicro Nano $_{\text {ano }} \cdot$ Micro

$\frac{\mathrm{dMéso}}{\mathrm{dt}}=\left(\right.$ rationMéso - égest $\left._{\text {Méso }}-\mathrm{excr}_{\text {Méso }}-\operatorname{mort}_{\text {Méso }}\right) \cdot$ Méso

$\frac{\mathrm{d} \text { Micro }}{\mathrm{dt}}=\left(\right.$ rationMicro - égest $_{\text {Micro }}-\mathrm{excr}_{\text {Micro }}-$ mort $\left._{\text {MésoMicro }}\right) \cdot$ Micro-rationMéso Micro $_{\text {. Méso }}$

$\frac{\mathrm{dN}_{\mathrm{det}}}{\mathrm{dt}}=\operatorname{mort}_{\text {Diat }} \cdot$ Diat mort $_{\text {Dino }} \cdot$ DinoN + mort $_{\text {Nano }} \cdot \mathrm{Nano}+$ relarg $_{\mathrm{N}} \cdot \mathrm{N}_{\text {ben }}+\left(\right.$ mort $_{\text {Méso }}+$ egest $\left._{\text {Méso }}\right) \cdot$ Méso

$+\left(\right.$ mort $_{\text {Micro }}+$ egest $\left._{\text {Micro }}\right) \cdot$ Micro $-\left(\mathrm{r}_{\min N}+\right.$ sédim $\left._{\text {det }}\right) \cdot \mathrm{N}_{\text {det }}-$ rationMicro det $\cdot$ Micro

$\frac{d S i_{\text {part }}}{d t}=$ mort $_{\text {Diat }} \cdot$ Diat + relarg $_{S_{i}} \cdot S_{i_{\text {ben }}}+$ rationMéso ${ }_{\text {Diat }} \cdot$ Méso $-\left(r_{\text {diss }}+\right.$ sédim $\left._{d e t}\right) \cdot S i_{\text {part }}$ 


$$
\begin{aligned}
& \frac{\mathrm{dP}_{\mathrm{det}}}{\mathrm{dt}}=\text { mort }_{\text {Diat }} \cdot \text { Diat }+ \text { mort }_{\text {Dino }} \cdot \text { DinoP }+ \text { mort }_{\mathrm{Nano}} \cdot \mathrm{Nano}+\text { relarg }_{\mathrm{P}} \cdot \mathrm{P}_{\text {ben }}+\left(\text { mort }_{\text {Méso }}+\text { egest }_{\text {Méso }}\right) \cdot \text { Méso }
\end{aligned}
$$

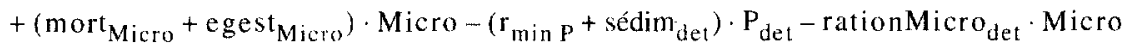

$$
\begin{aligned}
& \frac{\mathrm{dC}_{\mathrm{det}}}{\mathrm{dt}}=\text { mort }_{\text {Diat }} \cdot \text { Diat }+ \text { mort }_{\text {Dino }} \cdot \text { Dino }+ \text { mort }_{\text {Nano }} \cdot N \text { ano }+ \text { relarg }_{C} \cdot C_{\text {ben }}+\left(\text { mort }_{\text {Méso }}+\text { egest }_{\text {Méso }}\right) \cdot \text { Méso }
\end{aligned}
$$

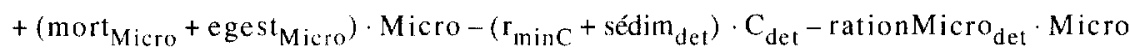

$$
\begin{aligned}
& \frac{\mathrm{dN}_{\text {ben }}}{\mathrm{dt}}=\text { sédim }{ }_{\text {Dial }} \cdot \text { Diat }+\operatorname{sédim}_{\mathrm{det}} \cdot \mathrm{N}_{\mathrm{det}}-\left(\mathrm{r}_{\text {min Nben }}+\text { relarg }_{N}\right) \cdot \mathrm{N}_{\text {ben }} \\
& \frac{\mathrm{dSi}_{\text {ben }}}{\mathrm{dt}}=\operatorname{sédim}_{\text {Diat }} \cdot \mathrm{Diat}+\operatorname{sédim}_{\mathrm{det}} \cdot S \mathrm{Si}_{\mathrm{det}}-\text { relarg }_{\mathrm{Si}} \cdot \mathrm{Si}_{\text {ben }} \\
& \frac{\mathrm{dP}_{\text {ben }}}{\mathrm{dt}}=\operatorname{sédim}_{\text {Diat }} \cdot \text { Diat }+ \text { sédim }_{\mathrm{det}} \cdot \mathrm{P}_{\mathrm{det}}-\left(\mathrm{r}_{\text {min Pben }}+\text { relarg }_{\mathrm{P}}\right) \cdot \mathrm{P}_{\text {ben }} \\
& \frac{\mathrm{dC}_{\text {benl }}}{\mathrm{dt}}=\operatorname{sédim}_{\text {Diat }} \cdot \text { Diat }+\operatorname{sédim}_{\mathrm{det}} \cdot \mathrm{C}_{\mathrm{det}}-\left(\mathrm{r}_{\text {minCben }}+\text { relarg }_{\mathrm{C}}\right) \cdot \mathrm{C}_{\mathrm{ben}} \\
& \mathrm{I}_{\text {min }} \quad: \text { taux de reminéralisation du matériel organique } \\
& \mu \quad \text { : taux de croissance du phytoplancton } \\
& \text { mort : taux de mortalité du phyto- et du zooplancton } \\
& \text { sédim : taux de sédimentation des diatomées et du matériel diétritique } \\
& \text { assim : taux d'assimilation des éléments nutritifs par les cellules de dinoflagellés } \\
& \text { ration, égest, excr : taux d'ingestion, d'égestion, d'excrétion du zooplancton } \\
& \text { relarg : taux de relargage du matériel benthique }
\end{aligned}
$$

Tous ces taux s'expriment en $\mathrm{j}^{-1}$. Par souci de simplicité, les rapports stoechiométriques constants entre les éléments (N/P/Si/C) ne sont pas figurés ici. Ces rapports s'appliquent aux concentrations en azote du phytoplancton et du zooplancton pour le calcul de $S i_{\text {diss }}$ et $P_{\text {min }}$ ainsi que $\mathrm{Si}, \mathrm{P}$ et $\mathrm{C}$ sous forme organique détritique. 